Fifth International Conference on Sustainable Construction Materials and

Technologies. http://www.claisse.info/Proceedings.htm

\title{
Conflicts in Design for Strengthening of Concrete Structures using Fibre-reinforced Polymer Composites
}

\author{
Kunal D Kansara $^{{ }^{*}}$ and Tim J Ibell ${ }^{2}$
}

\author{
${ }^{1}$ Senior Lecturer in Structural Engineering, Department of Civil Engineering, \\ Liverpool John Moores University, Liverpool L3 3AF, United Kingdom. \\ Email: K.D.Kansara@ljmu.ac.uk
}

${ }^{2}$ Professor of Structural Engineering, Department of Architecture and Civil Engineering, University of Bath, Bath BA2 7AY, United Kingdom.

Email: T.J.Ibell@bath.ac.uk

\begin{abstract}
The use of fibre-reinforced Polymer (FRP) composites as an externally-bonded tension reinforcement is an effective technique for the strengthening of under-performing concrete structures. The FRP-based strengthening design processes are significantly more conservative than the conventional norm in structural design. For example, the safety factors prescribed on FRP material properties could be up to 3 times as high than those typically prescribed on structural concrete. It is believed that such an approach will account for the considerably greater extent of uncertainties comprising of those inherent to the existing structure as well as those arising from the lack of knowledge and time-testimony of using FRP composites for structural strengthening. However, FRP composites involve substantially peculiar characteristics compared to steel when used as a tension reinforcement. Therefore, the design processes for strength (for new RC structures) and additional strength (for strengthening the existing $\mathrm{RC}$ structures) have conflicting design requirements and objectives. Thus, an approach of prescribing significantly higher factors of safety will not only be against the economic and environmental sustainability requirements, but can also fall short in providing the required safety margins and could instigate negative implications and undesirable side-effects under some design scenarios. This paper summarises important design conflicts arising from differential treatments in uncertainty, mechanics, failure mode, detailing and reliability management within the safety formats prescribed by various strengthening design standards. The implications of these conflicts, on the course and quality of strengthening design solutions, and that on their resultant safety-contents are discussed.
\end{abstract}

\section{INTRODUCTION}

Fibre Reinforced Polymer (FRP) composites are popularly used for the strengthening of under-performing members of existing concrete structures. An FRP-based 
strengthening system involves tactfully attaching FRP sheets or plies to the existing concrete member that act as externally bonded tension reinforcement.

The popularity of FRP composites is attributed mainly to their high strength-to-weight ratios and negligible susceptibility to corrosion. However, FRP composites exhibit some peculiar characteristics, which are in contrast with the traditional reinforcing material such as steel. Firstly, FRP composites exhibit directional orthotropy. Thus, their mechanical properties along and across the principal fibre directions are substantially different. Secondly, FRP composites don't offer any dependable plastic deformation at ultimate condition. Thirdly, an externally bonded FRP reinforcement involves a large possibility of premature failure through its debonding, which does not allow full mobilisation of their large rupture strain capacity. These peculiarities pose significant design challenges and concerns.

Additionally, due to the relatively short history of use within the construction industry, the long-term behaviour of FRP composites are less confidently known. Furthermore, the mechanics and response of externally bonded FRP are not fully known yet. Attributed to this lack of time testimony and knowledge, FRP-based structural strengthening involves a higher degree of uncertainty compared to the traditional structural materials. This forms an additional obligation for the strengthening design process and safety formats employed by the strengthening design standards.

This paper highlights some important design conflicts concerning the FRP-based strengthening of structures, and examines their implications on the strengthening design process and the quality of strengthening design solutions.

\section{EVOLUTION OF FRP-BASED STRENGTHENING DESIGN PROCESS}

Design process for FRP-based strengthening systems have evolved and refined over last few decades through research and applications. Seible (2001) presented a general scenario of strengthening design using FRP composites in civil structural environment, and Bank et al. (2001) provided a model specification for use of FRP for civil structures and covered a range of issues including material classification, scope of design specification and protocols for physical and mechanical material properties. Maruyama and Ueda (2001) and Fukuyama et al. (2001) discussed Japanese specifications on use of FRP for strengthening and retrofitting. Nanni (2001) provided a discussion on the North American design guidelines for FRP-based strengthening including the underlying principles, applications and some unresolved issues. Darby et al. (2007) presented a discussion on gaps in knowledge for strengthening of reinforced concrete structures using FRP composites. Darby et al. (2009) discussed the influence of changes in the cross-section on the effectiveness of strengthening schemes using externally bonded FRP. Ceroni and Pecce (2009) proposed design provisions for crack spacing and width in RC elements externally bonded with FRP. 
The focused research on developing strengthening design criteria led to the emergence of various design guidelines and standards for the design of FRP-based structural strengthening systems. Some of the most important international design guidelines include: ACI440-2R (2008) of the American Concrete Society (ACI), TR55 (2004) and TR55 (2012) of the Concrete Society, UK, FIB14 (2001) of the International Federation of Structural Concrete (FIB) and the design guidelines of Hong Kong [HKG (2010)], Japan [BRIJ (1998)] and Canada [ISIS (2001)]. All these design guidelines are conceptually similar to a large extent. Amongst these, ACI440 and TR55 are the two important international strengthening design guidelines prolifically followed worldwide.

The safety formats proposed by most strengthening design standards remain largely cloned from those proposed by the standards for design of new concrete structures. While most FRP-based strengthening design guidelines are conceptually similar to one another, they subscribe to different approaches for accounting for the uncertainties, addressing mechanics and ensuring reliability. The following sections discuss the design conflicts arising from:

- Uncertainty Management

- Mechanics Management

- Reliability Management

\section{UNCERTAINTY MANAGEMENT}

The uncertainty arising from the random variability in FRP material property values is addressed through statistical adjustment using the concept of characteristic values for these properties. The uncertainties arising from the variability in FRP material properties due to long-term environmental exposure and different methods for manufacturing and installing FRP are addressed through applying partial factors of safety on the characteristic values of FRP material properties to obtain their design values. The additional uncertainties associated with the use of FRP composites as an externally-bonded reinforcement are accounted for through a strength reduction factor prescribed on the nominal resistance contribution of the FRP reinforcement. Different strengthening design guidelines adopt the following different approaches on these counts, which lead to design conflicts:

- Unlike the conventional reduction of 1.64 times the standard deviation from the mean value of a material property to obtain a characteristic value with a customary $95 \%$ confidence interval, many FRP-based strengthening design guidelines prescribe higher reductions. For example, ACI440 and TR55 suggest a reduction of 3 and 2 times standard deviation respectively.

- FRP-based strengthening design guidelines invariably prescribe the partial factors of safety to account for long-term effect due to environmental exposure. 
However, only some design guidelines prescribe additional partial factors of safety on FRP material properties to account for the variation due to different manufacturing and installation methods used. For example, TR55 prescribe partial factors of safety accounting for environmental exposure as well as manufacturing and installation methods, whereas ACI440 choses to ignore the latter.

- Against the common convention of applying a partial factor of safety on the characteristic value of strength to obtain the corresponding design value, many strengthening design standards suggest applying partial factors of safety on one or more properties comprising the constitutive relationship for FRP. Accordingly, two distinct approaches are possible. Approach 1 (e.g. the one adopted by ACI440) includes applying a numerically identical partial factor of safety on the characteristic values of tensile strength and rupture strain capacity of FRP. In contrast with this, Approach 2 (e.g. the one adopted by TR55) involves applying numerically different partial factors of safety on modulus of elasticity and rupture strain capacity of FRP. With the FRP behaving linearly elastic right up to its rupture, the difference in applying partial safety factors on different material constitutive material properties can have conflicting implications.

- For flexural strengthening, amongst ACI440 and TR55 for example, only the former suggest a reduction factor on the flexural resistance contribution of the externally-bonded FRP reinforcement. TR55 does not prescribe any such factor.

- For shear strengthening, ACI440 proposes resistance reduction factor on the shear resistance contribution of externally bonded shear reinforcement depending upon if the shear strengthening configuration is either bond-critical (e.g., sides-only and U-wrapping configurations) or contact-critical (e.g., fullywrapped configuration). The bond-critical configurations involve a considerably higher reduction factor compared to the contact-critical configurations. TR55 does not prescribe any such reduction factor.

\section{CONFLICTS IN MECHANICS MANAGEMENT}

The post-strengthening failure modes are an important aspect of the flexural and shear strengthening design. Mechanics of FRP and steel reinforcements and concrete can influence the post-strengthening failure modes. Debonding, anchorage length and ductility are important elements of the mechanics of FRP-strengthened sections. Differences in design treatment of these elements can influence the design predictions for the governing failure modes in post-strengthening state.

Debonding. Debonding of externally-bonded FRP reinforcement from the concrete substrate leads to the premature loss of effectiveness of FRP before reaching its design rupture strain capacity. Within the design, debonding is popularly addressed through defining a strain limit for FRP $\left(\varepsilon_{\text {fd-debond }}\right)$ such that if the strain in FRP reinforcement 
exceeds this limit, it is deemed failed under debonding. Different approaches on this count leads to design conflict.

For flexural strengthening, some design guidelines [e.g., TR55, see Eq. (1)], arguably for the sake of simplicity, tend to prescribe a constant strain value for the debonding strain limit. In contrast, some design guidelines [e.g., ACI440, see Eq. (2)] subscribe to a more detailed mathematical model to arrive at debonding strain limit.

$\varepsilon_{\mathrm{fd}-\text { debond }}=0.008$

$\varepsilon_{\mathrm{fd} \text {-debond }}=0.41 \sqrt{\frac{f_{c}^{\prime}}{t_{\mathrm{FRP}} E_{\mathrm{FRP}}}} \leq 0.9 \varepsilon_{f d \text {-rupture }}$

In general, it can be perceived that the debonding strain limit criterion is relatively very stringent. Due to this, most flexural strengthening design solutions involving FRP failure are likely to be governed by failure of FRP under debonding and not in rupture.

For shear strengthening, on the other hand, most strengthening design guidelines refrain from prescribing a constant debonding strain limit. However, the approaches to arrive at the debonding strain limit value are considerably different. For example, see Eqs. (3) and (4) for TR55 and ACI440 respectively. While the approach of ACI440 appears simpler, the process of obtaining parameter $\kappa_{v}$ is relatively intricate.

$\varepsilon_{\mathrm{fd}-\text { debond }}=\kappa_{v} \varepsilon_{\mathrm{fd}-\text { rupture }}\left[\kappa_{v} \leq 0.75\right]$

$\varepsilon_{\text {fd-debond }}=0.64 \sqrt{\frac{0.18\left(f_{c u}\right)^{2 / 3}}{t_{\mathrm{FRP}} E_{\mathrm{FRP}}}}$

The possibility of FRP to fail through debonding is governed by the numerical values for the design debonding strain limit relative to that for the design rupture strain value. Kansara (2014) interpreted this as a failure mode switch. The failure mode switch for flexural strengthening is given by Eq. (8), while that for shear strengthening is given by Eq. (9).

$\varepsilon_{\mathrm{fd}}=\min \left[\varepsilon_{\mathrm{fd} \text {-rupture }} ; \varepsilon_{\mathrm{fd} \text {-debond }}\right]$

$\varepsilon_{\mathrm{fd}}=\min \left[\varepsilon_{\mathrm{fd}-\text {-rupture }} ; \varepsilon_{\mathrm{fd} \text {-debond }} ; \varepsilon_{\mathrm{fd} \text {-disintegration }} ; \varepsilon_{\mathrm{fd} \text {-fracture }}\right]$

It can be seen that, for shear strengthening, the competition to govern the failure of FRP relates to four strain limit criteria. Two of these correspond to the strain in FRP at which it ruptures $\left(\varepsilon_{\mathrm{fd}-\text {-rupture }}\right)$ and debonds $\left(\varepsilon_{\mathrm{fd}-\text { debond }}\right)$. The third strain limit criterion refers to a strain in FRP at which the concrete in the vicinity of the FRP shear reinforcement starts disintegrating $\left(\varepsilon_{\mathrm{fd} \text {-disintegration }}\right)$, which is often prescribed as a constant strain value (of the order of $0.4 \%$ ). In contrast with this, the fourth strain limit criterion refers to the strain in FRP dictating either elastic or rigid-body behaviours of 
regions on either sides of a shear crack $\left(\varepsilon_{\mathrm{fd} \text {-fracture }}\right)$, which is often prescribed as a fraction of design rupture strain capacity of the FRP. For example, Eqs. (10) and (11) present the strain limit $\varepsilon_{\mathrm{fd} \text {-fracture }}$ according to ACI440 and TR55 respectively.

$\varepsilon_{\mathrm{fd} \text {-fracture }}=0.75 \varepsilon_{\mathrm{fd} \text {-rupture }}$

$\varepsilon_{\mathrm{fd}-\text { fracture }}=0.50 \varepsilon_{\mathrm{fd}-\text { rupture }}$

It can also be seen that the debonding strain limits, such as the one presented through Eq. (1), involve an upper bound based on a fraction of design rupture strain. Such an upper bound, in effect, ceases the direct competition between the design rupture strain and design debonding strain limit. In such a case, a strengthening design solution involving failure of FRP at an ultimate limit state will never reach its full design rupture strain capacity. The ACI440 upper bound of $0.9 \varepsilon_{\mathrm{fd} \text {-rupture }}$ actually inflates the net partial factor of safety on rupture strain capacity by $10 \%$, which remains disguised to the strengthening designer.

It can be seen from the above that the differences in approaches in defining debonding strain limit can lead to numerically different design debonding strain values. Thus, the definition for the debonding strain limit can be used to 'tune' the competition between these strain limit criteria within the design of FRP-based strengthening systems. Furthermore, the practical implications of the debonding strain limits are that the rupture strain capacity of FRP over $0.4 \%$ for shear strengthening and that over $0.8 \%$ for flexural strengthening (with a constant strain value for debonding strain limit) are superfluous and of no structural advantage in design. This also puts a stringent constraint on the degree of strengthening that can be achieved using externally bonded FRP reinforcement.

It can also be seen that the differences in approach for defining strain limits related to debonding, concrete disintegration and fracture when seen alongside the value for design rupture strain (which depends up on the approaches and values adopted for prescribing the partial factors of safety), can influence the outcome of the failure mode switches. Thus, the differences in approaches for these aspects can lead to conflicting design predictions for the failure mode of FRP reinforcement.

Anchorage Length. The anchorage length $\left(L_{\mathrm{f}}\right)$ for the externally-bonded FRP reinforcement determines its effectiveness. Different strengthening design guidelines differ considerably on this count, which leads to design conflict.

For example, the TR55 anchorage length model, for flexural and shear strengthening, is presented through Eq. (12). The ACI440 model for flexural strengthening is very identical to this [Eq. (12)]. However, for shear strengthening, ACI440 proposes a different anchorage length model as shown in Eq. (14). 


$$
\begin{aligned}
& L_{\mathrm{f}}=0.7 \sqrt{\frac{t_{\mathrm{FRP}} E_{\mathrm{FRP}}}{f_{c t}}} \\
& L_{\mathrm{f}}=\sqrt{\frac{t_{\mathrm{FRP}} E_{\mathrm{FRP}}}{\sqrt{f_{c}^{\prime}}}} \\
& L_{\mathrm{f}}=\frac{23300}{\left(t_{\mathrm{FRP}} E_{\mathrm{FRP}}\right)^{0.58}}
\end{aligned}
$$

The inversed proportionality of $L_{\mathrm{f}}$ to $E_{\mathrm{FRP}}$ in Eq. (14) compared to Eqs. (12) and (13) can be noticed. It can be seen that the ACI440 doctrine of not applying any partial factors of safety on the modulus of elasticity is a conservative strategy for shear strengthening. However, for flexural strengthening their approach is non-conservative.

Ductility. An addition of the externally bonded FRP reinforcement for flexural strengthening can result into a considerable reduction in the sectional ductility in the post-strengthening state. This reduction depends, amongst many other conditions, on the externally bonded FRP content relative to the section size and the existing internal steel reinforcement content. Since the flexural resistance of a section and its sectional ductility are inversely proportional to each other, it is possible to compensate the drop in sectional ductility by imposing an additional penalty on the flexural resistance of the section. However, the differences in approach for prescribing this can lead to a design conflict.

For example, ACI440 proposes a capacity reduction factor to account for the reduction in post-strengthening sectional ductility in proportion to the strain in internal steel reinforcement in post-strengthening state. Unlike this, TR55 suggest a reduction of flat $15 \%$ in the flexural resistance of a strengthened section if the strain in internal steel reinforcement is less than a minimum required value. While such a flat reduction does account for the deviation in the sectional ductility between pre- and post-strengthening states, it doesn't offer a rational basis for such a reduction. This leads to a lesser control on the reliability content of a strengthened concrete section.

\section{CONFLICTS IN RELIABILITY MANAGEMENT}

Most strengthening standards follow limit state method (LSM) for the design of FRPbased strengthening systems. However, some of them have more explicit inclination towards probability-based reliability approaches while devising a safety format and various safety factors. For example, ACI440 follow Load and Resistance Factor Design (LRFD) approach, compared to TR55 which follows traditional LSM. Amongst many other differences between these two approaches, LRFD includes a specific resistance reduction factor to be applied on the post-strengthened flexural or shear resistance of the section to top-up or supplement the safety content to achieve the target reliability. The traditional LSM does not include this. While a choice of following either LRFD or traditional LSM approach is by no means is a conflict. However, TR55, for example, follows the traditional LSM and thus do not specify any strength reduction factors on this ground. However, in doing so it misses the 
opportunity to account for behavioural uncertainty which is in contrast the approach adopted by ACI440.

\section{IMPLICATIONS OF DESIGN CONFLICTS}

Kansara (2014) carried out a detailed assessment of the design conflicts in FRP-based structural strengthening design process. The implications of the design conflicts are summarised below:

Implications at Material Properties Level. Fig. 1 shows that under the approach of prescribing numerical identical partial factors of safety on rupture strain and tensile strength of FRP, the slope of stress-strain curve for FRP remains unchanged at mean, characteristic and design material property levels. Thus, the mean, characteristic and design values of modulus of elasticity of FRP remains numerically the same. This signifies the possible variability of modulus of elasticity (and hence the stiffness) of FRP do not get accounted for.

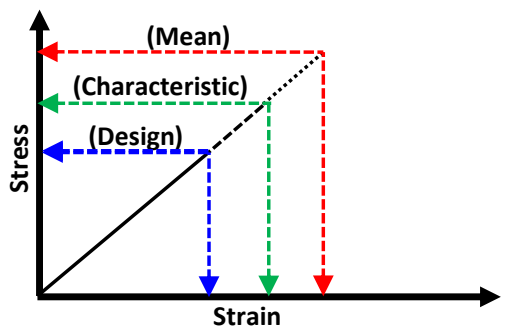

(a)

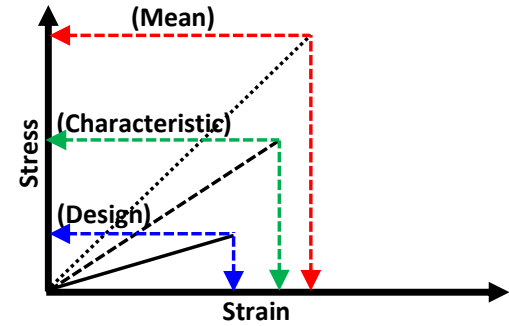

(b)

Fig. 1 Implications of design conflicts on the constitutive relationship of FRP

ACI440, while subscribing to this approach, does recognise this fact, but does not explain on the reasons. Only apparent explanation behind this could be that under certain circumstances not applying factors of safety on modulus of elasticity is a conservative approach. However, this is not true for all the situations.

Atadero and Karbhari (2009) explained that the treatment of modulus of elasticity of FRP in ACI440 poses a serious gap, particularly for confinement-based seismic retrofitting of columns, in which the design equations are most often directly based on the modulus of elasticity. In such cases, the variation in material property gets neglected.

The relative stringency of the equivalent partial factor of safety on the tensile strength of FRP accounting for the variability due to environmental exposure effect and qualitycontrol arising due to different manufacturing and installation techniques are presented through Fig. 2 and 3 respectively. 


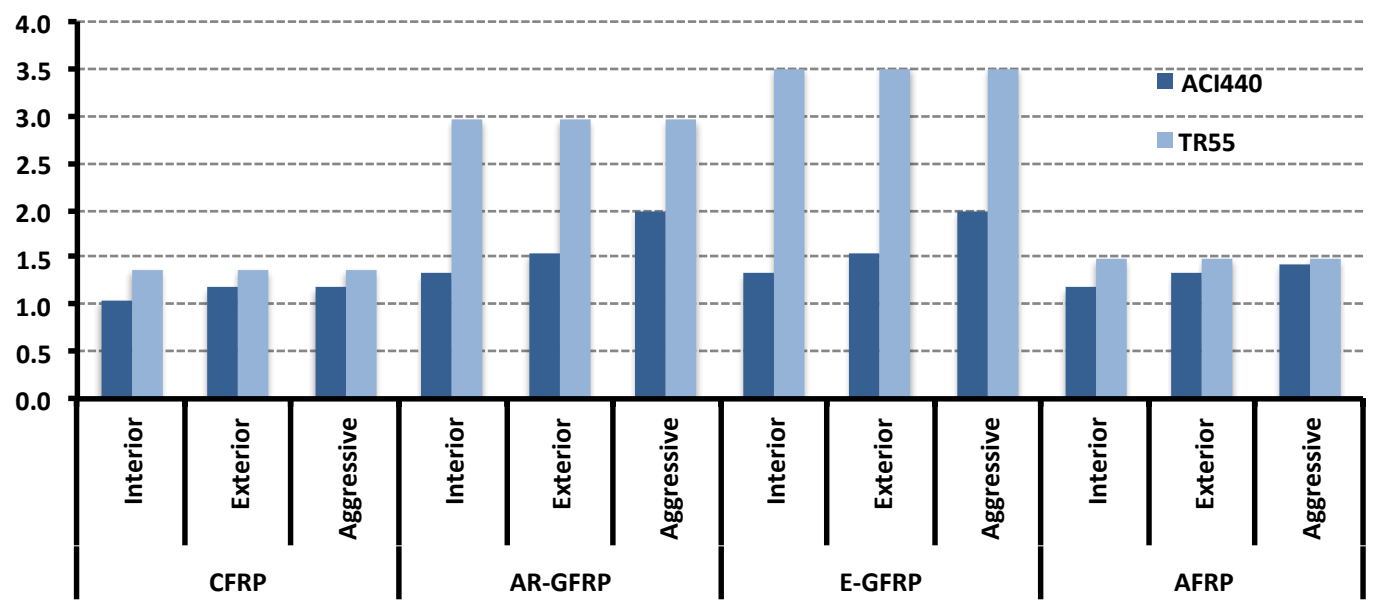

Fig. 2 Equivalent partial factor of safety on tensile strength of FRP accounting for environmental exposure effect

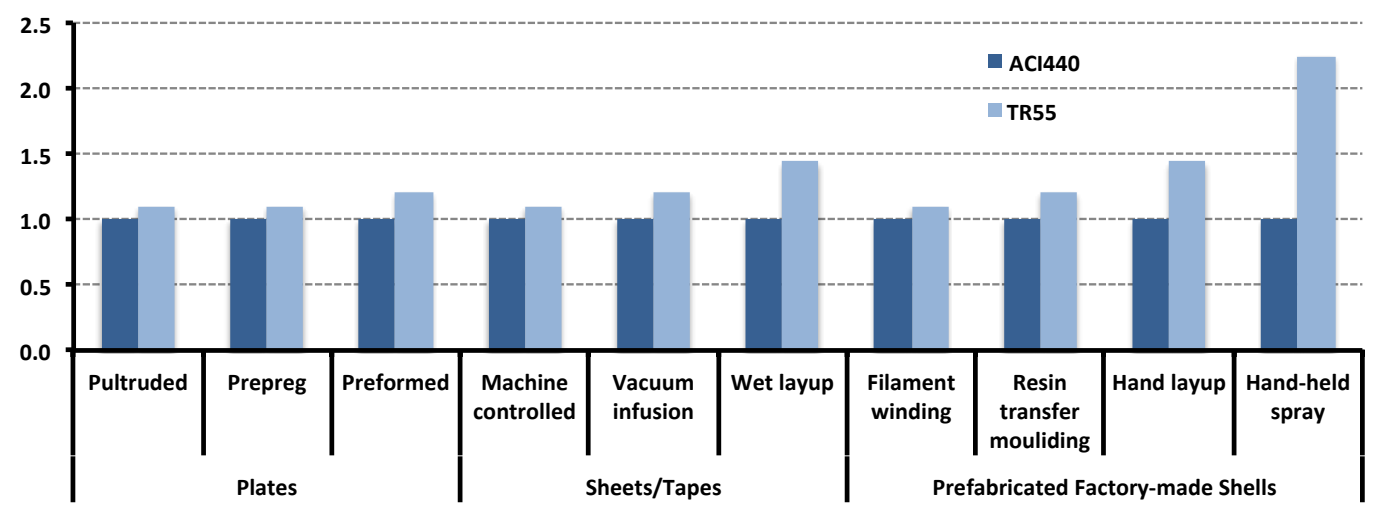

Fig. 3 Equivalent partial factor of safety on tensile strength of FRP accounting for quality variability due to different manufacturing and installation processes

Implications on Strengthening Design Process. Kansara (2014) proposed that the consequences of the design conflicts on the strengthening design process are twofold:

- Firstly, these conflicts can change the course of strengthening design process by influencing the design prediction for the failure modes. This could lead to a deviation between the design prediction of failure modes and the reality.

- Secondly, since different failure modes are differently sensitive to the partial factors of safety and that each failure mode carries fundamentally different characteristics, the deviation between the design predictions of failure modes and the reality can influence the quality of the strengthening design solutions and its actual safety content.

For example, Fig. 4 presents the possible flexural strengthening design solutions under identical conditions classified into three clusters according to their governing failure mode. Cluster A represents an over-reinforced strain-state in involves concrete 
crushing as the governing failure mode. Clusters B and C represent an Underreinforced strain-state, and involve FRP failure under rupture and FRP failure under debonding respectively. It can be seen that the changes in the extents of clusters under identical design scenarios, merely by the differences in values of the prescribed partial factors of safety on materials and different debonding strain limits.

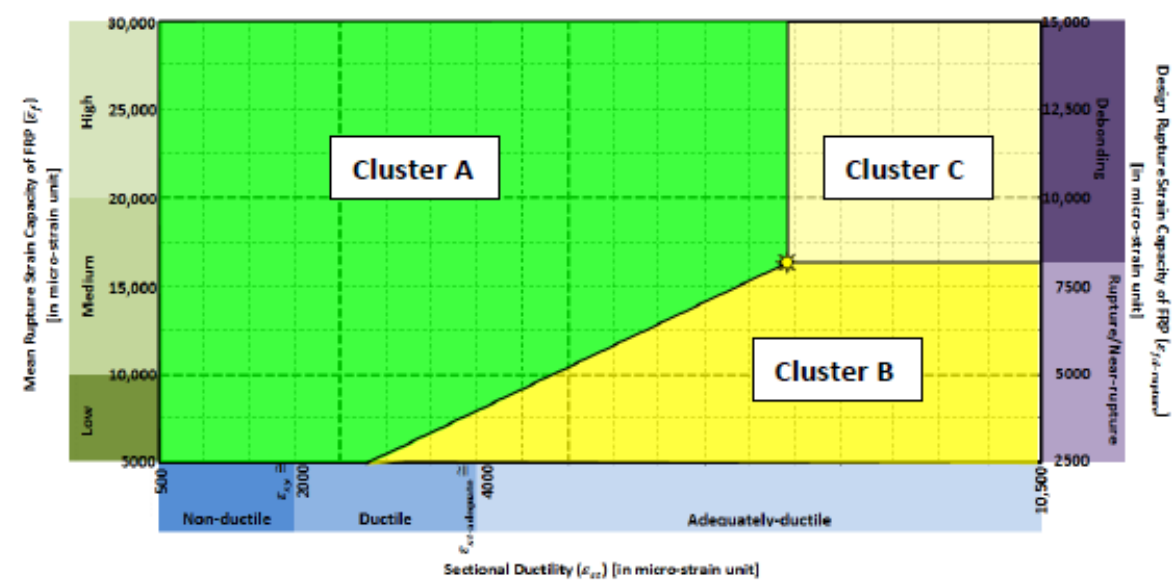

(a)

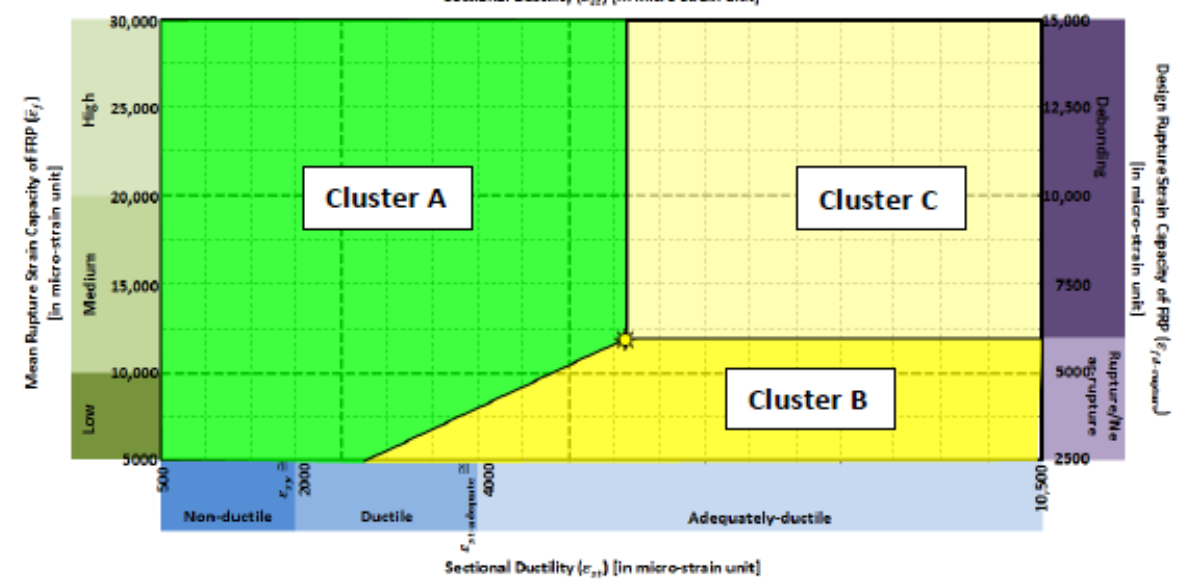

(b)

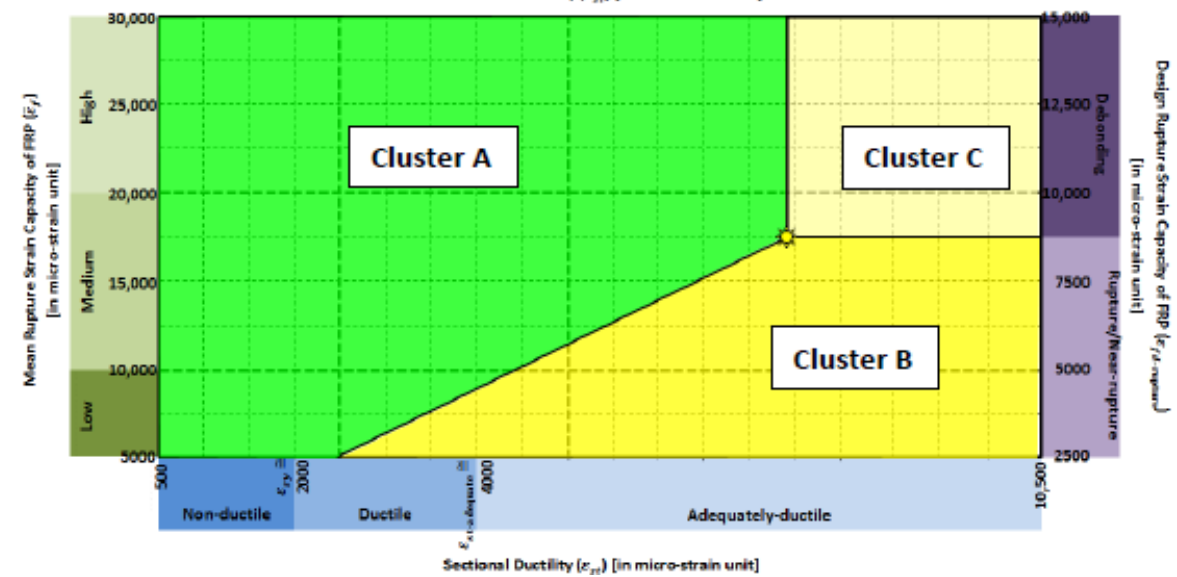

(c)

Fig. 4 Sensitivity of design predictions of failure modes in flexural strengthening for:

(a) $\varepsilon_{\mathrm{fd} \text {-debond }}=0.008$ and $\delta_{\mathrm{f} \varepsilon}=1$; (b) $\varepsilon_{\mathrm{fd} \text {-debond }}=0.006$ and $\delta_{\mathrm{f} \varepsilon}=1$; and (c) $\varepsilon_{\mathrm{fd} \text {-debond }}=0.006$ and $\delta_{\mathrm{f} \varepsilon}=0.9$ 
Fig. 5 shows the variation in post-strengthening flexural resistance $\left(M / b d^{2}\right)$. The horizontal axis on these plots represents sectional ductility in form of strain in the internal steel reinforcement.
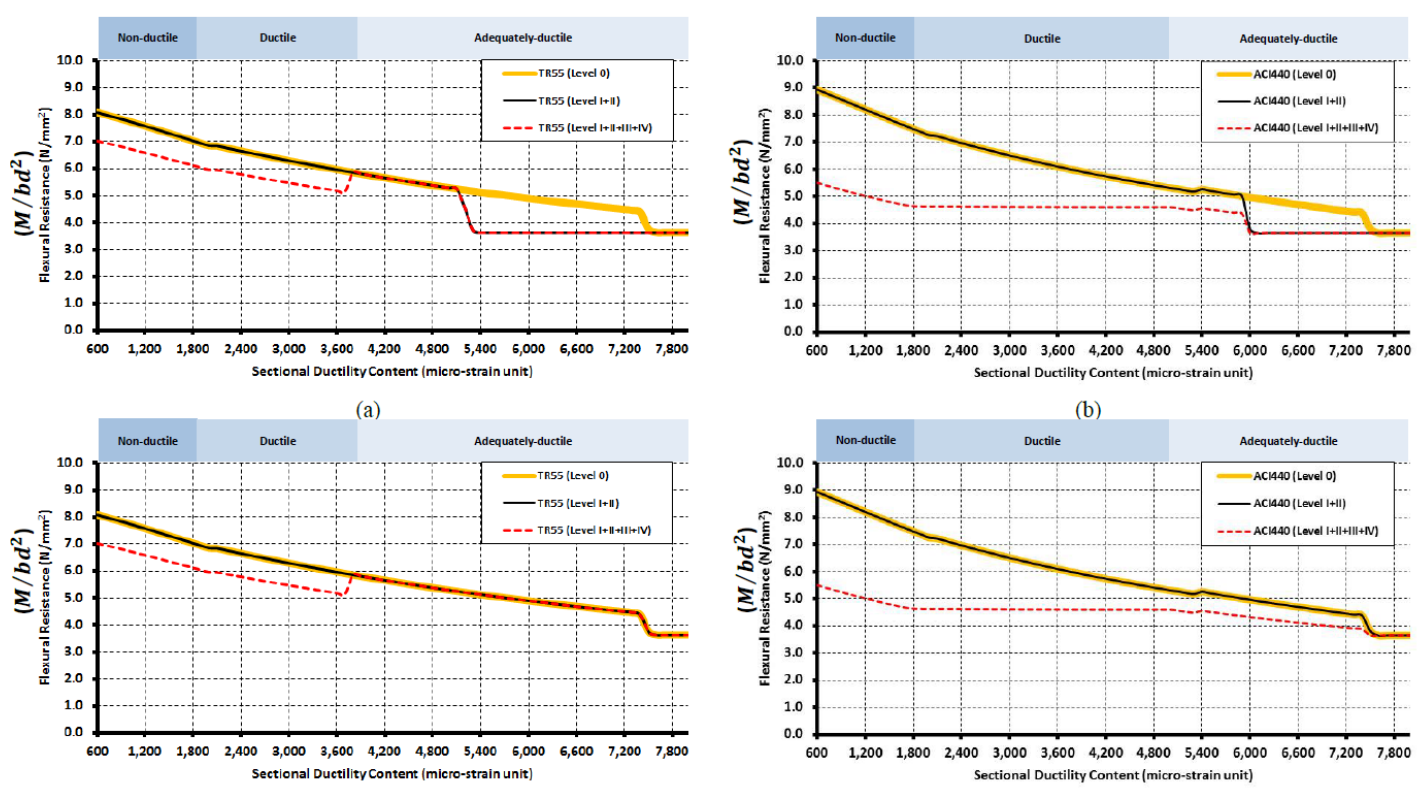

(c)

(d)

Fig. 5 Flexural resistance variation plots for: (a) and (b) involving Concrete failure or FRP rupture; and (c) and (d) involving Concrete failure or FRP debonding

For given identical circumstances, a particular value of sectional ductility represents a unique flexural strengthening design solution, and thus a range of sectional ductility represented by the horizontal axis represents a range of possible design solutions under a given design scenario. Combination (Level 0) indicates omission of all the partial factors of safety on FRP material properties and resistance reduction factors. Combination (Level I+II) considers the applicable partial factors of safety on FRP material properties only, and ignores the resistance reduction factors. Combination (Level I+II+III+IV), on the other hand, considers the applicable partial factors of safety on FRP material properties as well as applicable resistance reduction factors. The flexural strengthening design solutions with lesser sectional ductility (initial range of horizontal axis) typically involve concrete failure, while the rest represents FRP failure, which could be either under rupture or debonding. The difference between the Combinations (Level 0) and (Level I+II) represents the safety-content obtained due to the applicable partial factors of safety on FRP materials, and that between Combinations (Level 0) and (Level I+II+III+IV) represents the safety-content obtained due to the applicable partial factors of safety on FRP material properties as well as on resistance reduction factors.

It can be seen that for the strengthening design solutions involving concrete failure carry safety-content compared to those involving FRP rupture. This is despite of 
concrete failure mode being completely insensitive to the applicable partial factors of safety on FRP. The only resistance reduction factor prescribed by both ACI440 and TR55 to account for the lack of sectional ductility comes to the rescue here. Between the failure modes involving FRP failures, the ones involving rupture do include some safety-content, while the ones involving debonding do not reflect any safety content.

This shows that the reliability indices for different failure modes will be substantially different. Between ACI440 and TR55, it can be seen that the former generally results into higher safety-content compared to the latter. This is despite the fact that the partial factors of safety on FRP material properties for ACI440 are less stringent than those for TR55. The TR55 approach of not prescribing any resistance reduction factors is the reason attributed for this fall in safety-content.

Fig. 6 presents the variation in post-strengthening shear resistance $\left(V_{\mathrm{FRP}}\right)$, normalised with a parameter $\left(H_{0}\right)$ description of which is not directly relevant to the discussion here. This variation is for the possible shear strengthening design solutions for sidesonly, U-wrapping and fully-wrapped configurations under identical circumstances. It can be seen that post-strengthening shear resistance values for ACI440 and TR55 vary substantially for identical conditions, reflecting that the safety-content and reliability indices will be substantially different for these two strengthening design standards.
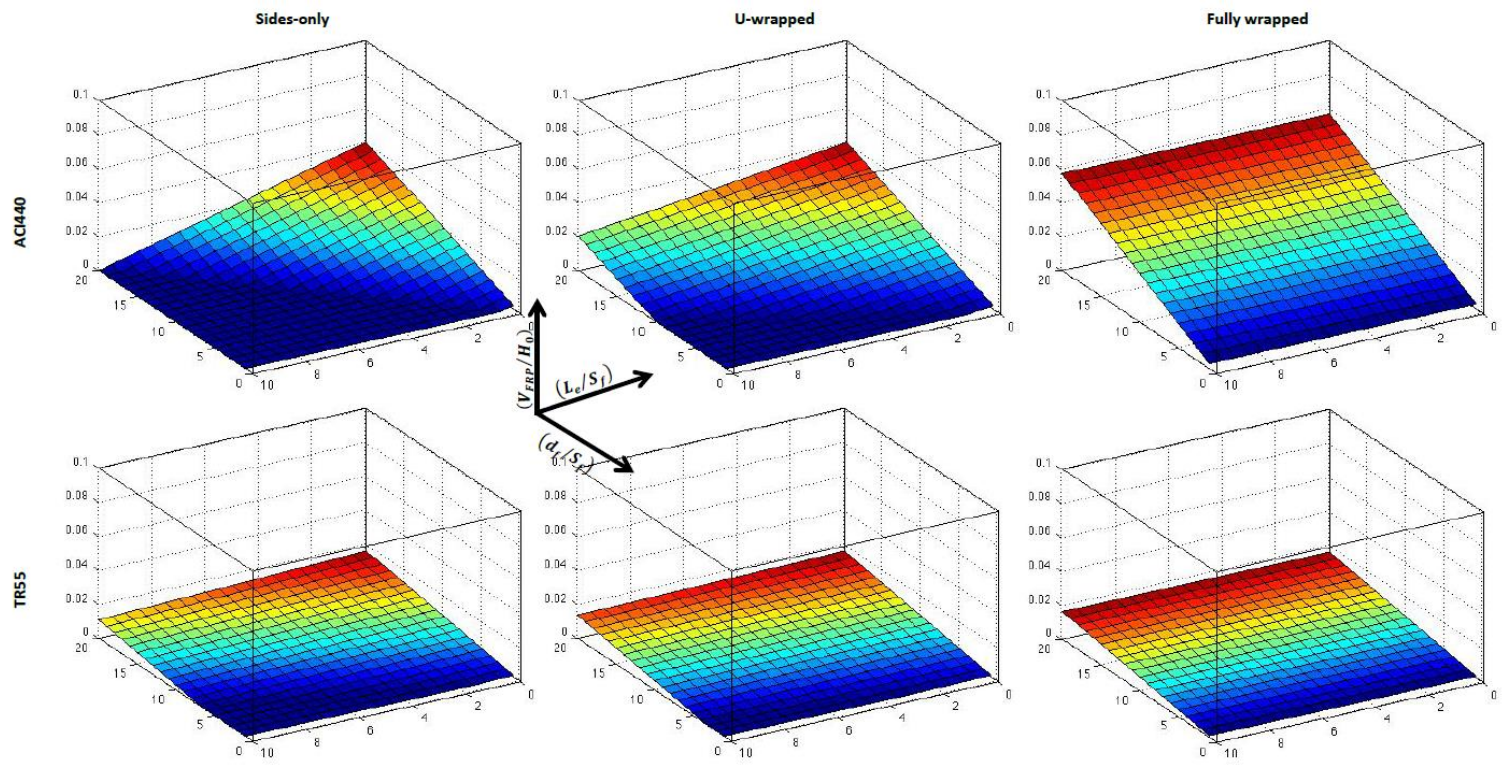

Fig. 6 Variation in shear strengthening design solutions under identical conditions

\section{SUMMARY AND CONCLUSIONS}

A range of design conflicts arising from different treatment of uncertainties, mechanics, failure modes, detailing and reliability management approaches are 
presented in this paper. The implications of the differences within the safety formats used by different strengthening design standards under these design conflicts are evidently demonstrated. In particular, it is shown that the different approaches and varying degree of stringency in prescribing partial factors of safety and resistance reduction factors affect the course and the quality of strengthening design solutions, and can lead to a deviation between the design prediction for the governing failure modes and the reality. This, in turn, affects the net safety-content associated with the strengthening design solutions and consequently the reliability in the poststrengthening state. Clearly, these conflicts and their implications need to be accounted for while revising the existing strengthening design standards.

\section{REFERENCES}

Seible, F.: Designing with FRP composites in civil structural environment, in FRP composites in construction (Ed. Teng, J-G.) (2001).

Bank, L. et al: A model specification for FRP composites for civil engineering structures, in FRP composites in construction (Ed. Teng, J-G.) (2001).

Maruyama, K. and Ueda, T.: JSCE recommendations for upgrading of concrete structures with use of continuous fibre sheets, in FRP composites in construction (Ed. Teng, J-G.) (2001).

Fakuyuma, H. et al: Japanese design and construction guidelines for building retrofit using FRP composites, in FRP composites in construction (Ed. Teng, J-G.) (2001).

Nanni., A.: North American guidelines for concrete reinforcement and strengthening using FRP composites, in FRP composites in construction (Ed. Teng, J-G.) (2001).

Darby, A. et al: Gaps in current knowledge for FRP strengthening of RC structures, In: Proceedings of advanced composites in construction, Bath, UK (2007).

Darby et al.: Influence of changes in cross sections on the effectiveness of the externally bonded FRP strengthening, Journal of composites for construction, 13(3), pp. 208-216 (2009).

Ceroni, F. and Pecce, M.: Design provisions for crack spacing and width in $R C$ elements externally bonded with FRP, Composites Part B: Engineering, 40(1), pp. 1728. 